\title{
Contributions of category and fine-grained information to location memory: When categories don't weigh in
}

\author{
Marcia L. Spetch, Alinda Friedman, Jared Bialowas, and Eric Verbeek \\ University of Alberta, Edmonton, Alberta, Canada
}

\begin{abstract}
Several studies have shown that people's memory for location can be influenced by categorical information. According to a model proposed by Huttenlocher, Hedges, and Duncan (1991), people estimate location by combining fine-grained item-level information in memory with category-level information. When the fine-grained information is inexact, category-level information is given greater weight, which leads to biased responses. We manipulated the distribution of locations presented in order to alter the usefulness of category information, and we manipulated background texture in order to alter accuracy of fine-grained memory. The distributional information reduced bias without altering overall accuracy of responding, whereas the background texture manipulation affected accuracy without changing bias. Our results suggest that category information may weigh in only when it is actively processed.
\end{abstract}

Memory for places can be critical to the survival of humans and nonhumans alike. Not surprisingly, the underlying mechanisms and the factors that influence location memory have been the focus of extensive research in cognitive science, developmental psychology, animal behavior, and neuroscience. One common finding is that spatial memory rarely relies on a single source of information; rather, multiple sources of spatial information are often encoded (Cheng, Shettleworth, Huttenlocher, \& Rieser, 2007; Huttenlocher, Hedges, \& Duncan, 1991; Spetch \& Kelly, 2006). Understanding how multiple cues are integrated to control spatial search or estimation is an important step in the study of spatial memory.

Cheng et al. (2007) suggested that the integration of spatial information may be considered in terms of a functional Bayesian framework. According to their framework, spatial information from multiple cues is likely to be combined, as long as the information is not too discrepant among the different cues along the relevant dimension(s). They suggested that information from multiple cues is sometimes combined in a near optimal fashion, using a weighted average. According to Bayesian principles, the optimal weight for each cue should be proportional to the variance of the spatial information encoded by that cue. Cheng et al. (2007) proposed that a weighted combination of information may occur when spatial estimation is based on two or more sources of current information, on current information together with prior information, or on current information together with categorical information. The latter situation is the focus of our investigation.

Huttenlocher and colleagues conducted a series of studies to investigate how location memory for specific items is influenced by spatial categories (e.g., Huttenlocher, Hedges, Corrigan, \& Crawford, 2004; Huttenlocher et al., 1991; Huttenlocher, Newcombe, \& Sandberg, 1994). In one of their tasks (Huttenlocher et al., 1991), people were shown a series of dots within a circular space and were required to reproduce the dots' locations from memory. Although the dots were randomly distributed throughout the circle, people showed a bias to remember them as having been located toward the midpoint of each of four quadrants formed by imagined horizontal and vertical lines through the center of the circle. Huttenlocher et al. (1991) suggested that people naturally categorize the space into these four quadrants and that a dot's location is represented not just in terms of fine-grained metric information, but also in terms of the spatial category (i.e., the quadrant) in which it was located. Both types of information are assumed to be nonbiased, and there is evidence suggesting that fine-grained information and categorical information are weighted independently (e.g., Hund \& Plumert, 2002). However, bias is introduced during retrieval and reproduction, when the fine-grained item information is combined with the category information. The locational center of the quadrant is assumed to be the central value of the category and, hence, the locational prototype. Thus, averaging item information with the category prototype leads to a bias of responses to the center of the quadrant. The contribution of category information is assumed to improve performance overall, but it also leads to an observed systematic response bias (Huttenlocher et al., 1991). Consistent with a Bayesian interpretation, the bias increases when the fine-grained location memory is degraded by an interference task during retention (Huttenlocher et al., 1991). An

M. L.Spetch, mspetch@ualberta.ca 
interference task should reduce certainty about the finegrained item location and, hence, result in greater weight being given to the category prototype.

The basic finding, that people show biases in location memory toward the center of quadrants in a circular space, has been replicated several times (e.g., Laeng, Peters, \& McCabe, 1998; Verbeek \& Spetch, 2008). Biases in location memory with other geometric spaces (ellipse, triangle, square, and pentagon) have also been found (Wedell, Fitting, \& Allen, 2007). Wedell et al. found that shape affected the spacing of the prototypes, but in all spaces, people still appeared to divide the space into four quadrants based on the vertical and horizontal axes. Thus, a viewer-based division of space based on up, down, left, and right appears to provide a strong natural categorization scheme for 2-D spaces presented in the frontalparallel plane. Moreover, Huttenlocher et al. (2004) found that the bias in a circular space remained even when the distribution of dots was skewed toward the cardinal axes so that no dots appeared near the center of the quadrants. In fact, even showing participants this skewed distribution of dots, or having them categorize the dots according to the skewed distribution, failed to eliminate the bias. One possible reason for the failure of their manipulation to eliminate bias is that Simmering and Spencer (2007) found that it is difficult for people to imagine or impose arbitrary category boundaries without perceptual support, even when instructed. The spatial category boundaries that Simmering and Spencer used were arbitrary in that they were not easily aligned with reference frames within either the body or the environment. In contrast, the gravitational vertical is always available as a reference frame, and verticality per se is easy to process, in perception (even when the head is tilted; Friedman \& Hall, 1996), imagery (Franklin \& Tversky, 1990), and proprioception. Furthermore, there are many vertical and horizontal cues that can help form a reference frame in a normal indoor testing environment (e.g., sides of a computer monitor, corners between walls, surface of a desk, etc.). All of these internal and external cues may make imagining vertical and horizontal category boundaries both nonarbitrary and relatively easy. Hence, the categorization of circular space into four quadrants based on horizontal and vertical symmetry may be a strong natural tendency.

The biases shown in circular space are not immune to all manipulations. For example, Verbeek and Spetch (2008) found that biases were altered when visible dividing lines were placed within the circular space; in these cases, people showed biases toward the lines, rather than toward the category centers. Verbeek and Spetch suggested that, when visible dividing lines were present, people may have used the lines as landmarks for encoding the dots' locations. This landmark-based encoding scheme may have replaced or supplemented the encoding of categorical information. A related possibility is that the lines provided perceptual anchors that were explicit and exact and, thus, as effective as verticality is in an undivided space.

The present experiments followed the functional approach of Cheng et al. (2007) and explored manipulations designed to separately alter the weight given to fine- grained and categorical information. One of our manipulations altered the distribution of the dots in an attempt to reduce the value of remembering categorical information. Specifically, whereas the control group was tested with dots from all quadrants in the circle, the participants in the experimental group received dots located in only a single quadrant. For the control group, remembering which quadrant a given dot was in on each trial should be beneficial, because it would narrow the range of possible response locations to the remembered quadrant; we therefore should observe the usual bias toward the center of each of the four quadrants, because the category information will weigh in to the response. In contrast, for the quadrant group, the continued presentation of the dots within a single quadrant should make trial-by-trial encoding of the category information redundant. Specifically, once the participants learned that the dots always fell within a single quadrant, there would be no need to specifically attend to and remember quadrant information on a trial-by-trial basis. If category information weighs in only when it is actively held in short-term memory, the quadrant group should show less bias to the center of the category than the control group.

Although our prediction that the dots' distribution should alter bias seems inconsistent with the results of Huttenlocher et al. (2004), there is some precedence for predicting that stimulus distributions affect category bias. For example, in a size estimate task, Duffy, Huttenlocher, and Crawford (2006) found that children's responses were biased toward the central value of the stimulus distribution, as predicted by a Bayesian model of category effects on estimation, and that the pattern of bias was sensitive to the dispersion of the stimulus distribution. For location estimates in particular, Spencer and Hund (2002, Experiment 3) found some evidence that the distributions of targets within a large rectangular space altered biases. In their task, dot reproduction was imbedded within a "spaceship game" in which participants were told to remember the location of target spaceships. Each person was tested with three target locations. When the targets were presented in both halves of the space, people showed a bias away from the midpoint and toward the center of each half of the space. When the targets were shown in only one half, biases toward the centers of each half of the space still occurred, but responses were also systematically influenced by the distribution of the three targets within that half of the space. However, their task differed from ours in a number of ways, including their use of a small number of locations per participant and accuracy feedback after each response.

Our second manipulation was designed to alter the accuracy of encoding of the fine-grained location information. In Experiment 1, we attempted to disrupt the encoding of fine-grained information for some participants (the background group) by presenting the circle and the dot against a textured background and then removing the texture during dot reproduction (see Figure 1). We expected that removing the background texture would disrupt location memory if the participants attempted to use the background (or the part of it that was near the dot) as a land- 
Background Group, Experiment 1

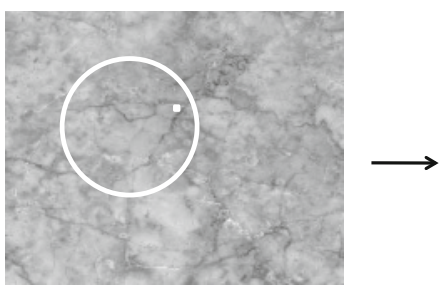

Encoding Phase

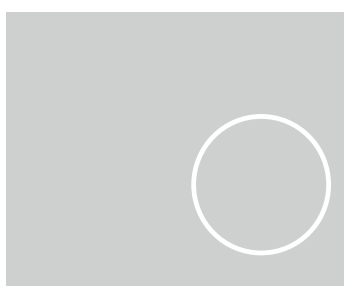

Response Phase

Figure 1. Schematic diagram showing an example of a trial for the participants in the background group in Experiment 1.

mark to encode the fine-grained metric location of the dot. For example, if a participant attempted to use a particular edge within the texture as a landmark or beacon and tried to remember the dot location in terms of this cue, location memory should be reduced, because the texture was not present during reproduction. We therefore predicted that absolute response accuracy would be reduced for the background group, relative to the control group. On the other hand, bias produced by averaging the item memory with category information should be maintained, or even higher, in the background group, because the reduction in certainty about item information caused by removing the background during reproduction should result in greater weight being given to the category information.

In Experiment 2, the dot was presented against a textured pattern that was fixed within the circle during the encoding phase (see Figure 4). For some participants (the absent group), the texture was removed during the reproduction phase, so we expected to see a disruption of accuracy but no decrease in bias. For other participants (the present group), the texture remained in the circle during the reproduction phase. We expected that the presence of local texture cues at both encoding and reproduction might facilitate the encoding of fine-grained dot location. For example, when the same texture is present in the circle during both the encoding and the reproduction phases, using a part of the texture near the dot as a landmark or beacon might improve the accuracy of item location memory. To the extent that fine-grained location encoding is more certain, reliance on category information should diminish. We therefore expected to see an increase in accuracy and a decrease in bias for the present group, relative to the absent group.

\section{EXPERIMENT 1}

This experiment included three groups of participants: a control group, a quadrant group, and a background group. The control group was given a standard dot location task in which they briefly saw a dot appear somewhere in a circle and then tried to reproduce the dot's location by clicking within a blank circle. The location of the circle on the screen moved between the encoding and reproduction parts of the trial. This meant that the participants were required to remember the location of the target dot relative to the circle, and not relative to themselves or the location on the computer screen. This procedure had been found to produce the typical bias toward the center of quadrants by a control group (empty circle condition) in a previous study (Verbeek \& Spetch, 2008).

Across trials, the dot locations were evenly distributed throughout the circle. The quadrant group received the same procedure, except that dot locations always fell within a single quadrant for each participant (with the specific quadrant varying across participants). The procedure for the background group was the same as that for the control group, except that the circle and dot were placed against a textured background during the encoding phase. The texture was removed from the background during the reproduction part of the trial. The specific background texture varied across participants in this group.

\section{Method}

Participants and Design. The participants were 36 student volunteers from introductory psychology classes at the University of Alberta. Gender was not recorded. The participants received partial course credit for participating. The sessions lasted about $20 \mathrm{~min}$. Twelve participants were assigned in random order to each of three groups: control, background, and quadrant.

Apparatus and Procedure. The experiment was conducted in a small quiet room that contained two desks with computers that faced opposite walls. The participants were tested singly or in pairs facing away from each other. The stimuli were presented on 17-in. CRT monitors with screen resolution set to $1,280 \times 1,024$ pixels. The stimuli consisted of a $1.5-\mathrm{mm}$ square yellow dot and a $0.25-\mathrm{mm}$ yellow line that formed a stimulus circle with a diameter of approximately $15 \mathrm{~cm}$. The stimulus circle was presented in a randomly selected location on the screen, and the dot appeared in 1 of 69 evenly distributed positions on a notional grid within the circle; the positions were approximately $16 \mathrm{~mm}$ vertically and $16 \mathrm{~mm}$ horizontally apart from each other. This resulted in 13 possible dot locations within each quadrant, 1 dot location at the exact center of the circle, and 8 dot locations on each of the horizontal and vertical axes. The 13 dot locations within each quadrant resulted in 9 unique angular locations within each quadrant (there were 2 dot locations that fell at the 3 rd and 7 th angular location, and 4 dot locations fell at the center angular location in each quadrant). This distribution of dots followed exactly the procedure described by Huttenlocher et al. (1991). The circle and dot were presented either against a solid gray background (control and quadrant groups) or against one of three gray textures (background group). The textures consisted of clouds, leaves, or a marble pattern. 
For the background and control groups, each of the 69 dot positions was presented once, with 13 dots falling within each quadrant. The remaining 17 dots consisted of 1 dot at the exact center of the circle and 8 dots on each of the horizontal and vertical axes. This followed the procedure used by Huttenlocher et al. (1991). For the quadrant group, all 69 dots appeared in only one quadrant, with each of the 13 critical dot locations in that quadrant presented four times. The center dot was again presented once, and each of the 8 dot locations that fell on the horizontal and vertical borders of the quadrant was presented twice. Three randomly selected participants were assigned to each quadrant. For the background group, each participant saw only a single type of texture (clouds, leaves, or marble), with four randomly selected participants assigned to each texture condition.

At the beginning of the session, the participants were instructed that their task was to remember the location of a dot within a circle and that they would need to mouse-click in an empty circle to indicate where they remembered seeing the dot. Each trial consisted of a study/encoding phase followed by a response/reproduction phase. For the encoding phase, the circle was presented alone for $1.5 \mathrm{sec}$, and then the stimulus dot was added for $1.5 \mathrm{sec}$. The screen was then cleared for a 1-sec delay. During the reproduction phase, the stimulus circle appeared in a new, randomly selected screen location, and a crosshair controlled by a mouse was presented at the center of the screen. For the background group, the textured background was present during encoding but was changed to a gray slide for the reproduction phase, as shown in Figure 1. Responses made during the first $1 \mathrm{sec}$ of the response phase were not registered, to avoid counting impulsive responses, and responses that fell outside of the circle were also ignored. A click within the circle after $1 \mathrm{sec}$ caused the crosshairs to disappear, and a dot was displayed for $1 \mathrm{sec}$ at the location that the participant clicked (this was designed to provide visual feedback that a response had been registered). A 6-sec interval with a black screen separated the trials.

Data analysis. On each trial, the $x$ - and $y$-coordinates of the dot location, the mouse click, and the center of the circle were recorded. Because the circle moved on the screen between encoding and reproduction, all locations were computed relative to the center of the circle. These locations were used to derive measures of bias and accuracy.

To measure bias, the locations were converted to polar coordinates. For example, when the target location is converted to polar coordinates $(r, \theta), r$ is the distance between the target and the 0,0 origin at the center of the circle, and $\theta$ is the angle between the $x$-axis and the vector from the origin to the target location. Angular error (bias) is computed as the signed error between the $\theta$ s for the target and the response locations. A systematic bias in signed errors toward the centers of the four quadrants is typically observed in the location estimate paradigm and is taken as an indication of the heavier weighting of category information.

To determine whether signed error showed a systematic bias, we first averaged across any radial distances from the center of the circle that had the same angle. This resulted in nine angles that had the same relative positions in each quadrant. We excluded the data from trials on which the dot fell on an axis or at the center, because these dots did not fall within a particular quadrant. We then pooled the data across quadrants by renumbering each quadrant to start at angle $0^{\circ}$, so that each quadrant had dots at angles of $14^{\circ}, 18^{\circ}, 27^{\circ}$, $34^{\circ}, 45^{\circ}, 56^{\circ}, 63^{\circ}, 72^{\circ}$, and $76^{\circ}$, based on clockwise rotation. This pooling was conducted within subjects for the participants in the control and background groups and between subjects for those in the quadrant group. As per Huttenlocher et al. (1991), our analysis of bias excluded trials on which the response was more than $45^{\circ}$ away from the correct location in either direction (this accounted for less than $2 \%$ of the data).

To examine response accuracy (i.e., how closely the participants' responses were to the target location), we used the $x$ - and $y$-coordinates of the target location and its estimate to calculate the Euclidean distance between the two, separately for each trial.
Euclidean distance provides an indication of the accuracy of finegrained metric information, which could vary independently of angular bias. In both this experiment and Experiment 2, we also analyzed angular response error (calculated as the unsigned angular deviation between the target and response), with and without the exclusion of trials used for the angular bias analyses. These analyses yielded the same overall pattern of results as our analyses of distance error. Because the analyses of both angular error and distance error converged on the same conclusions, we report only Euclidean distance as our measure of response accuracy.

For the participants in the background group, preliminary analyses revealed no significant effects of background type on either bias or accuracy (all $p \mathrm{~s}>.1$ ), and therefore, the data were averaged across the three background conditions.

\section{Results}

Figure 2 shows bias (angular deviation) for each group as a function of angle within a quadrant, and Figure 3 shows accuracy (in terms of mean distance error) for each group. In general, the quadrant manipulation altered bias but not accuracy, whereas the background manipulation altered accuracy but not bias.

Bias. The data of interest for bias were angular response deviations as a function of angular dot location. To the extent that the participants used categorical information to reproduce the dot location, we expected that responses would be biased toward the center of each quadrant. As a result, a plot of angular bias scores against angle within the quadrant should show a monotonically decreasing function, with bias changing from positive to negative around the midpoint of the quadrant. The slope of this bias function should be reduced by any manipulation that

\section{Experiment 1}

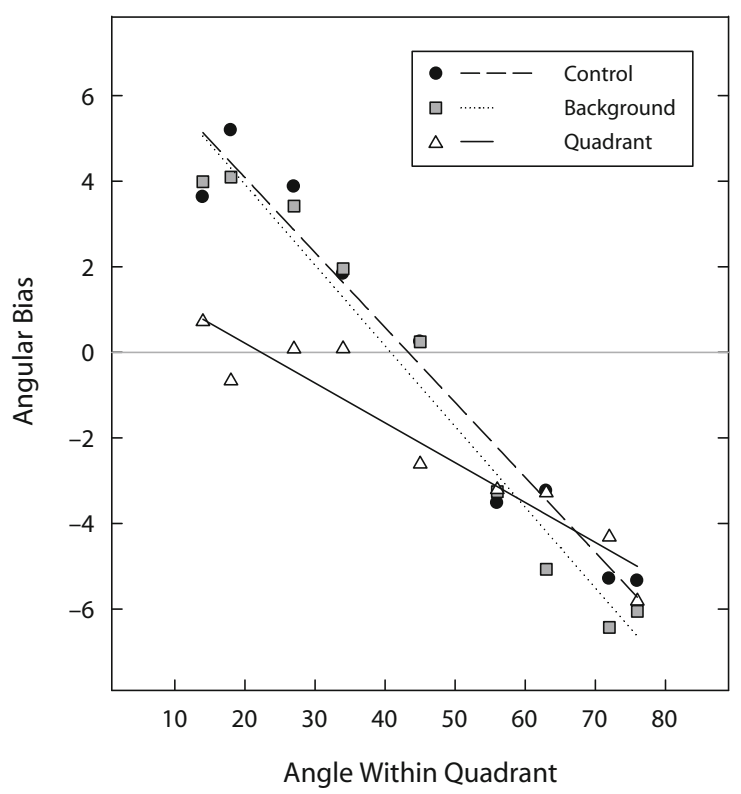

Figure 2. Angular bias as a function of angle within quadrants for the participants in the control, background, and quadrant groups in Experiment 1. 


\section{Experiment 1}

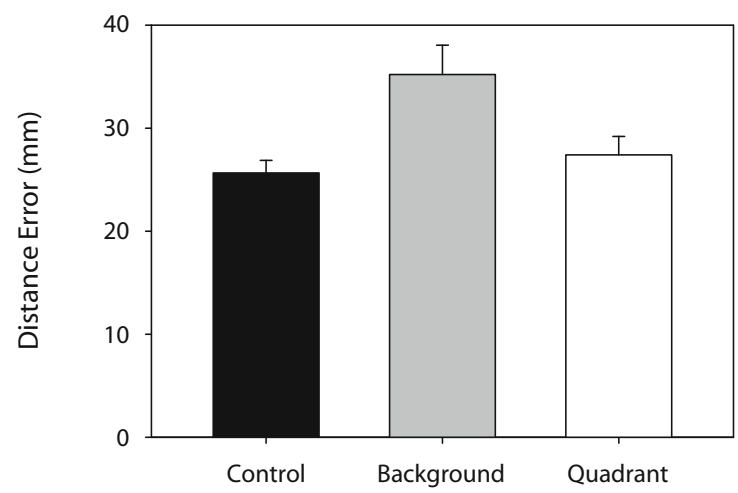

Figure 3. Euclidean distance error for the participants in the control, background, and quadrant groups in Experiment 1. Error bars are standard errors of the means.

resulted in less weighting of category information or more weighting of fine-grained information.

The angular bias scores were submitted to a group (control, background, and quadrant $) \times$ angle $\left(14^{\circ}, 18^{\circ}, 27^{\circ}\right.$, $34^{\circ}, 45^{\circ}, 56^{\circ}, 63^{\circ}, 72^{\circ}$, and $\left.76^{\circ}\right)$ mixed ANOVA. There was a main effect of angle $\left[F(8,264)=45.16, M S_{\mathrm{e}}=10.113\right.$, $\left.\eta_{\mathrm{p}}^{2}=.578\right]$ and a group $\times$ angle interaction $[F(16,264)=$ $\left.2.29, M S_{\mathrm{e}}=10.113, \eta_{\mathrm{p}}^{2}=.122\right]$. Furthermore, both the linear and cubic trends were reliable for the main effect of angle $\left[F(1,33)=148.92, M S_{\mathrm{e}}=23.26, \eta_{\mathrm{p}}^{2}=.819\right.$, for the linear trend; $F(1,33)=17.06, M S_{\mathrm{e}}=8.23, \eta_{\mathrm{p}}^{2}=.341$, for the cubic trend]. The interactions between the linear and cubic trends and group were also significant $[F(2,33)=$ $5.48, M S_{\mathrm{e}}=23.26, \eta_{\mathrm{p}}^{2}=.249$, for the linear $\times$ group interaction; $F(2,33)=3.30, M S_{\mathrm{e}}=8.23, \eta_{\mathrm{p}}^{2}=.167$, for the cubic $\times$ group interaction]. These trends and, particularly, their interactions with group indicate that the form of the bias functions differed by group. The cubic trend may be consistent with Fitting, Wedell, and Allen's (2007) fuzzy boundary extension of the category adjustment model; however, we were not explicitly testing their model here. By inspection of Figure 2, it appears that the quadrant group was primarily responsible for the interactions; subsequent analyses (described below) of both the slopes and the correlations between the actual angle and the estimated bias supported this claim.

The mean slopes for the control, background, and quadrant groups were $-.189,-.175$, and -.095 , respectively. The slopes were significantly different from zero for all groups $[t(11)=9.55,7.37$, and 4.92 for the control, background, and quadrant groups, respectively; all $p \mathrm{~s}<.001]$. Importantly, and in accord with our prediction, the slopes differed significantly between the groups $[F(2,33)=$ $\left.5.66, M S_{\mathrm{e}}=0.005, \eta_{\mathrm{p}}^{2}=.255\right]$. Post hoc analyses using the Bonferroni correction showed that the slope for the quadrant group was significantly different from the slopes for the other two groups, which did not differ. The significant polynomial trends, as well as the difference in slopes and correlations between the quadrant group and the other groups, converge on the conclusion that the quadrant group's response function was different from those of the other groups.

To test the possibility that the smaller slope for the quadrant group might result from the additional exposures to each specific location and, hence, a refinement in fine-grained item encoding, we compared the slopes for the quadrant group for the first and second halves of the session and for the first and last quarters of the session. These slopes were small in all cases, and there were no significant differences between the slopes for the first half $(-.094)$ versus the last half $(-.078)$ of the session $[t(11)=0.60, p>.5]$ or for the first quarter $(-.079)$ versus the last quarter $(-.073)$, of the session $[t(11)=$ $0.197, p>.5]$. An analysis of the Fisher-transformed correlations between the actual and remembered angles (in polar coordinates) for the control, background, and quadrant groups yielded a reliable main effect $[F(2,33)=$ 9.80, $\left.M S_{\mathrm{e}}=0.204, \eta_{\mathrm{p}}^{2}=.373\right]$. Post hoc analyses using the Bonferroni correction showed that the correlation for the quadrant group differed from the correlations for the other two groups, which did not differ from each other. The back-transformed mean correlations for the control, background, and quadrant groups were $-.955,-.884$, and -.561 , respectively.

Accuracy. The Euclidean distance between the response and correct locations (in millimeters) was higher for the background group (35.21) than for the control $(25.67)$ or quadrant $(27.42)$ groups $[F(2,33)=6.04$, $\left.M S_{\mathrm{e}}=51.28, \eta_{\mathrm{p}}^{2}=.268\right]$. Post hoc analyses using the Bonferroni correction confirmed that response error for the background group was higher than that for either the control group or the quadrant group, which did not differ from each other (see Figure 3). Thus, the background manipulation reduced the overall accuracy of the location estimate.

\section{EXPERIMENT 2}

Experiment 1 showed a clear effect of dot distribution on bias. The participants in both the control and background groups showed a strong bias toward the center of the four quadrants. By contrast, the participants in the quadrant group showed a much smaller bias, suggesting that they weighted category information less than did the participants in the other groups. The repetition of the dots in the quadrant condition did not appear to be the reason for the reduced bias, because there was no significant change in slope from the first to the second half of testing or from the first to the last quarter of testing. Interestingly, the reduced bias for the quadrant group was not accompanied by a significant change in accuracy.

In contrast to the effect of dot distribution, our background texture manipulation had no significant effect on bias. However, the background group showed lower accuracy than did the other two groups, suggesting that either the background sometimes interfered with coding of the target location or removing the background in the response circle may have interfered with responding by in- 
creasing uncertainty. Experiment 2 provided a replication of the effects of dot distribution and further explored the role of background texture. In particular, in Experiment 2, we compared accuracy when the background was present during both encoding and reproduction versus when it was present only during encoding, as in Experiment 1. We expected that the participants who saw the dot against a textured background during encoding but then reproduced the dot against a blank background would show a reduction in response accuracy, as was seen in Experiment 1. However, we expected that the participants who saw the dot against a texture during encoding and then reproduced the dot location with the texture still present and in the same place within the circle might be able to use texture cues as beacons or landmarks to facilitate memory of the dot location. Thus, we expected that response accuracy would not be reduced in this condition and might even be enhanced, relative to control accuracy.

\section{Method}

Participants and Design. The participants were 53 volunteer students ( 25 females) from introductory psychology classes at the University of Alberta. They received partial course credit for participating. The sessions lasted about $20 \mathrm{~min}$. The participants were assigned in random order to each of four groups: control $(n=14)$, quadrant $(n=12)$, present $(n=13)$, and absent $(n=14)$.

Apparatus and Procedure. The apparatus was the same as that in Experiment 1, except that a 17-in. LCD monitor was used in place of the CRT monitor. For the control and quadrant groups, the procedure was identical to that described in Experiment 1. For the present and absent groups, the procedure was identical to that for the control group, except for the presence of a marble texture within the circle. For both the present and absent groups, the texture filled the circle during presentation of the dot. During the subsequent reproduction phase, in which the circle was presented in a new loca- tion on the computer monitor, the circle retained the exact texture for the participants in the present group, but the texture was absent and replaced by the solid gray background color for the participants in the absent group (see Figure 4).

The data were filtered and averaged as described in Experiment 1.

\section{Results}

Figure 5 shows the angular bias scores for each group as a function of angle within a quadrant, and Figure 6 shows mean distance error for each group. In general, the quadrant manipulation altered bias but not response error. As in Experiment 1, bias was not affected by the background manipulation, but the participants who saw the background during encoding only (the absent group) showed the highest response error.

Bias. The angular bias data were submitted to a group (control, absent, present, and quadrant) $\times$ angle $\left(14^{\circ}, 18^{\circ}\right.$, $27^{\circ}, 34^{\circ}, 45^{\circ}, 56^{\circ}, 63^{\circ}, 72^{\circ}$, and $76^{\circ}$ ) mixed ANOVA. There was a main effect of angle $\left[F(8,392)=43.85, M S_{\mathrm{e}}=9.33\right.$, $\left.\eta_{\mathrm{p}}^{2}=.472\right]$ and a group $\times$ angle interaction $[F(24,392)=$ $\left.2.90, M S_{\mathrm{e}}=9.33, \eta_{\mathrm{p}}^{2}=.151\right]$. Furthermore, both the linear and cubic trends were again reliable for the main effect of angle $\left[F(1,49)=105.93, M S_{\mathrm{e}}=27.76, \eta_{\mathrm{p}}^{2}=\right.$ .684 , for the linear trend; $F(1,49)=41.41, M S_{\mathrm{e}}=7.21$, $\eta_{\mathrm{p}}^{2}=.458$, for the cubic trend]. And again, the interactions between group and the linear and cubic trends were significant $\left[F(3,49)=6.08, M S_{\mathrm{e}}=27.76, \eta_{\mathrm{p}}^{2}=.272\right.$, for the linear $\times$ group interaction; $F(3,49)=3.57, M S_{\mathrm{e}}=$ $7.21, \eta_{\mathrm{p}}^{2}=.179$, for the cubic $\times$ group interaction], indicating that the form of the functions differed by group. By inspection of Figure 5, it can be seen that the quadrant group was again primarily responsible for these interactions; subsequent analyses of both the slopes and the cor-

Absent Group, Experiment 2



Present Group, Experiment 2
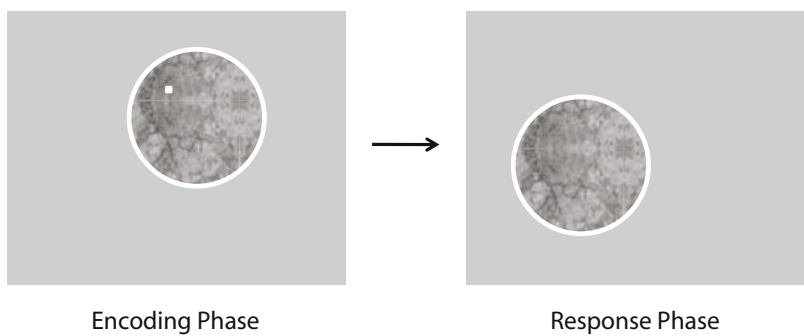

Figure 4. Schematic diagram showing an example of a trial for the participants in the absent and the present groups in Experiment 2. 


\section{Experiment 2}

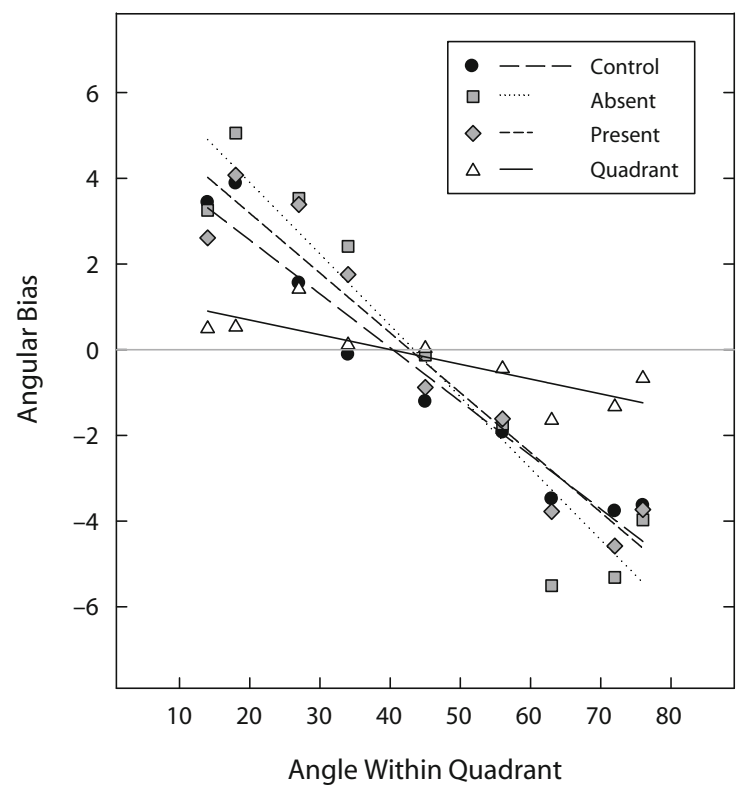

Figure 5. Angular bias as a function of angle within quadrants for the participants in the control, absent, present, and quadrant groups in Experiment 2.

relations between the actual angle and the estimated bias again supported this claim.

The mean slopes for the control, absent, present, and quadrant groups were $-.125,-.168,-.138$, and -.035 , respectively $\left[F(3,49)=6.22, M S_{\mathrm{e}}=0.007, \eta_{\mathrm{p}}^{2}=.276\right]$. Post hoc analyses using the Bonferroni correction showed that the slope for the quadrant group was significantly different from the slopes for the other three conditions, which did not differ from each other. The slope was significantly below zero for the control group $[t(13)=5.15$, $p<.001]$, the absent group $[t(13)=8.22, p<.001]$, and the present group $[t(12)=5.77, p<.001]$ but was not significantly different from zero for the quadrant group $[t(11)=1.77, p>.1]$

As in Experiment 1, the smaller slope for the quadrant group did not seem to result from the additional exposures to each specific location and, hence, a refinement in item encoding, because the slopes for the quadrant group did not differ between the first half $(-.045)$ and the second half $(-.038)$ of the session $[t(11)=0.38$, $p>.5]$ or between the first quarter $(-.052)$ and the last quarter $(-.013)[t(11)=1.65, p>.10]$. In addition to the analysis of the slopes, analysis of the Fisher-transformed correlations between actual and estimated angles yielded a reliable main effect of group $\left[F(3,49)=5.84, M S_{\mathrm{e}}=\right.$ $\left.0.374, \eta_{\mathrm{p}}^{2}=.263\right]$. Post hoc analyses using the Bonferroni correction showed that the correlation for the quadrant group differed from the correlations for the other three groups, which did not differ from each other. The backtransformed mean correlations for the control, absent, present, and quadrant groups were $-.760,-.848,-.799$, and -.296 , respectively.

Accuracy. Response accuracy was lowest for the participants in the absent group. The Euclidean distances between response and target location for the control, absent, present, and quadrant groups were 26.4, 30.7, 23.8, and $21.9 \mathrm{~mm}$, respectively $\left[F(3,49)=4.19, M S_{\mathrm{e}}=46.4, \eta_{\mathrm{p}}^{2}=\right.$ .204]. Post hoc analyses using the Bonferroni correction showed that distance error for the absent group was higher than that for the quadrant group; no other differences were significant.

\section{DISCUSSION}

The distribution of locations presented to the participants clearly affected response bias in a manner predicted by a functional Bayesian approach to location memory. Specifically, when all of the locations to be remembered came from a single quadrant, bias toward the center of the quadrant was reduced (Experiment 1) or eliminated (Experiment 2). This is consistent with the functional predictions that category information should be useful only when the category to which items belong is variable and/or when the fine-grained information is uncertain or nonexistent. When all the information to be remembered belongs to a single category, the category in which the dot was located on the current trial is redundant rather than informative, and thus, it may not be necessary to actively remember or use category information. Certainly, in this type of situation, the category-level information would be expected to receive less weight than when it is informative. As such, a tendency to show a bias toward the category prototype should be reduced or absent.

The robust effect of distribution on bias was not accompanied by a significant effect on reproduction accuracy: Mean response error for the quadrant group was slightly higher than control error in Experiment 1 and lower than control error in Experiment 2; in neither case was the

\section{Experiment 2}

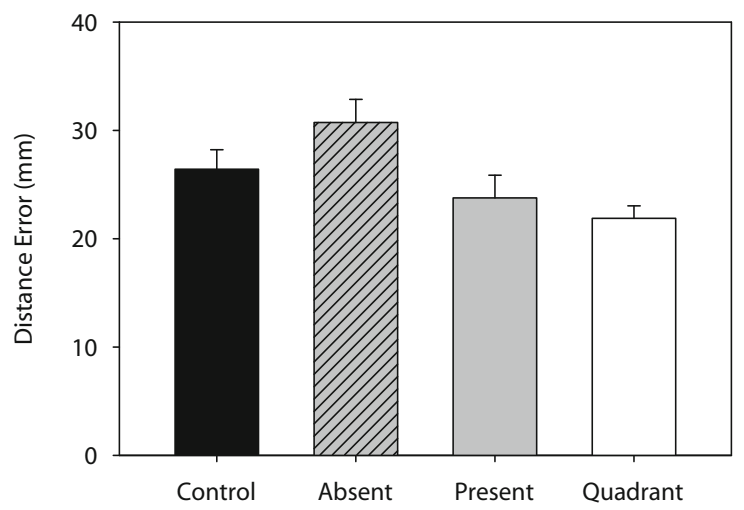

Figure 6. Euclidean distance error for the participants in the control, absent, present, and quadrant groups in Experiment 2. Error bars are standard errors of the means. 
difference significant. This finding, that accuracy in the quadrant group was not significantly different from accuracy in the control group, is interesting because, from a resource allocation perspective, eliminating the need to encode category information by making it redundant might be expected to improve the ability to encode finegrained item information. The apparent independence of accuracy and bias suggests that the encoding of category information is independent of the encoding of finegrained location information. This is consistent with the model proposed by Huttenlocher et al. (1991) and with evidence from Hund and Plumert (2002). Interestingly, the independence between levels has also been found to hold for global-scale location estimates (Friedman, 2009; Friedman \& Montello, 2006).

The effects of our background texture manipulation were also consistent with the independence of category and fine-grained memory. In this case, however, the manipulation altered response accuracy without affecting angular bias. The background manipulations had no significant effects on bias in either experiment. In Experiment 1, the background group, which had a patterned texture during encoding and a blank texture during reproduction, was significantly less accurate in their responses than either the control group or the quadrant group. In Experiment 2, the absent group, which saw the dot against a patterned texture during encoding but saw a blank texture during reproduction, showed the lowest accuracy among the four groups, although the absent group was significantly different only from the quadrant group. In contrast, the present group, which saw the same patterned texture during both encoding and reproduction, showed accuracy very similar to that of the control and quadrant groups. Thus, as was expected, accuracy was not reduced in this case, but our hypothesis that this manipulation would enhance memory for the fine-grained location information was not supported. Taken together, it appears that the presence of a background during encoding and its removal during reproduction disrupted response accuracy; however, bias was unaffected by this manipulation.

As was discussed by Cheng et al. (2007), the combining or averaging of independent sources of information to generate a response appears to occur in many domains and across many species. In fact, responding sometimes reflects an integration of different modalities of information, such as visual and haptic cues (e.g., Ernst \& Banks, 2002), or different types of information, such as space and time (Cheng, Spetch, \& Miceli, 1996). At other times, information from multiple sources may compete, with one source of information dominating at the expense of the other (e.g., Chamizo, 2003; Spetch, 1995). Functional considerations may help to explain when and why integration occurs. For example, Cheng et al. (2007) suggested that in spatial navigation, integration of cues is more likely to occur with small discrepancies between the different sources of information; if the discrepancy is too large, it becomes statistically unlikely that the cues are pointing to the same place or object, and hence, one cue should be selected, rather than averaging of the information. In line with this expectation, Friedman, Spetch, and Ferrey (2005) found that for pictures of objects, pigeons could integrate over relatively small angles, but not over larger ones; Friedman and Waller (2008) found similar results for integration over different perspectives of scenes. Similarly, Gepshtein, Burge, Ernst, and Banks (2005) found that combining visual and haptic information depends on their spatial separation. They suggest that the likelihood that two signals come from the same object is reduced by spatial separation and, therefore, the mechanism of visual-haptic combination is specialized for signals coming from the same place.

Our main finding, that altering the distribution of locations presented reduced (Experiment 1) or eliminated (Experiment 2) bias toward the centers of spatial categories, suggests that the integration of fine-grained location information with category information is not an automatic or obligatory process. It is important to note that this reduction in bias occurred without changing the perceptual support in any way; in particular, nothing differed visually between the quadrant and control groups. It is also important that the reduction in bias occurred without significantly altering the accuracy of responding. Although it is possible that the reduction in bias occurred because the participants in the quadrant group recategorized the experimental quadrant into smaller parts, inspection of the bias functions for the quadrant group did not show a clear pattern that would be suggestive of a new division within the quadrant. If a recategorization of the quadrant space occurred, it appeared to differ across participants. Thus, a simpler interpretation of the bias results is that the quadrant group was less influenced by category information than were the other groups.

From a functional perspective, category information should still be useful for participants in the quadrant group, insofar as they still need to know into which of the four categories to place their response. However, actively retaining the category information in short-term memory on a trial-by-trial basis should not be necessary. It is possible that the participants encoded and actively retained the categorical information on each trial but did not integrate it with fine-grained location information when making a response decision. Alternatively, it may be that a bias toward the prototype of the spatial category occurs only when the category information is actively held in short-term memory or is required to be retrieved from long-term memory, which was not the case for the quadrant group in either experiment. The latter possibility seems consistent with the suggestion that category-induced biases occur during reconstruction from memory, rather than during perception (Crawford, Huttenlocher, \& Engebretson, 2000). In either case, our results suggest that biases resulting from the combination of category and item information may occur only when category-level information is actively processed and weighs in to the response decision.

\section{AUTHOR NOTE}

This research was supported by NSERC research grants to M.L.S. and A.F. We thank Alisha Brown, Kirsten Williams, and Maria Modanu for their help in data collection, and Bernd Kohler for assistance with programming. Correspondence concerning this article should be addressed to M. L. Spetch, Department of Psychology, University of Alberta, Edmonton, AB, T6G 2E9 Canada (e-mail: mspetch@ualberta.ca). 


\section{REFERENCES}

Chamizo, V. D. (2003). Acquisition of knowledge about spatial location: Assessing the generality of the mechanism of learning. Quarterly Journal of Experimental Psychology, 56B, 102-113. doi:10.1080/ 02724990244000205

Cheng, K., Shettleworth, S. J., Huttenlocher, J., \& Rieser, J. J. (2007). Bayesian integration of spatial information. Psychological Bulletin, 133, 625-637. doi:10.1037/0033-2909.133.4.625

Cheng, K., Spetch, M. L., \& Miceli, P. (1996). Averaging temporal duration and spatial position. Journal of Experimental Psychology: Animal Behavior Processes, 22, 175-182. doi:10.1037/0097 $-7403.22 .2 .175$

Crawford, L. E., Huttenlocher, J., \& Engebretson, P. H. (2000). Category effects on estimates of stimuli: Perception or reconstruction? Psychological Science, 11, 280-284. doi:10.1111/1467-9280.00256

Duffy, S., Huttenlocher, J., \& Crawford, L. E. (2006). Children use categories to maximize accuracy in estimation. Developmental Science, 9, 597-603. doi:10.1111/j.1467-7687.2006.00538.x

ERNST, M. O., \& BANKS, M. S. (2002). Humans integrate visual and haptic information in a statistically optimal fashion. Nature, 415, 429-433.

Fitting, S., Wedell, D. H., \& Allen, G. L. (2007). Memory for spatial location: Cue effects as a function of field rotation. Memory \& Cognition, 35, 1641-1658.

FrankLin, N., \& TVersky, B. (1990). Searching imagined environments. Journal of Experimental Psychology: General, 119, 63-76. doi:10.1037/0096-3445.119.1.63

FriEdMAN, A. (2009). The role of categories and spatial cuing in globalscale location estimates. Journal of Experimental Psychology: Learning, Memory, \& Cognition, 35, 94-112. doi:10.1037/a0013590

Friedman, A., \& HaLl, D. L. (1996). The importance of being upright: Use of environmental and viewer-centered reference frames in shape discriminations of novel three-dimensional objects. Memory \& Cognition, 24, 285-295.

Friedman, A., \& Montello, D. R. (2006). Global-scale location and distance estimates: Common representations and strategies in absolute and relative judgments. Journal of Experimental Psychology: Learning, Memory, \& Cognition, 32, 333-346. doi:10.1037/0278 $-7393.32 .3 .333$

Friedman, A., Spetch, M. L., \& Ferrey, A. (2005). Recognition by humans and pigeons of novel views of 3-D objects and their photographs. Journal of Experimental Psychology: General, 134, 149-162. doi:10.1037/0096-3445.134.2.149

FrIedMAN, A., \& WALLER, D. (2008). View combination in scene recognition. Memory \& Cognition, 36, 467-478. doi:10.3758/MC.36.3.467
Gepshtein, S., Burge, J., Ernst, M. O., \& Banks, M. S. (2005). The combination of vision and touch depends on spatial proximity. Journal of Vision, 5, 1013-1023.

Hund, A. M., \& Plumert, J. M. (2002). Delay-induced bias in children's memory for location. Child Development, 73, 829-840. doi:10.1111/1467-8624.00441

Huttenlocher, J., Hedges, L. V., Corrigan, B., \& Crawford, L. E. (2004). Spatial categories and the estimation of location. Cognition, 93, 75-97. doi:10.1016/j.cognition.2003.10.006

Huttenlocher, J., Hedges, L. V., \& Duncan, S. (1991). Categories and particulars: Prototype effects in estimating spatial location. Psychological Review, 98, 352-376. doi:10.1037/0033-295X.98.3.352

Huttenlocher, J., Newcombe, N., \& Sandberg, E. H. (1994). The coding of spatial location in young children. Cognitive Psychology, 27, 115-147. doi:10.1006/cogp.1994.1014

Laeng, B., Peters, M., \& McCabe, B. (1998). Memory for locations within regions: Spatial biases and visual hemifield differences. Memory \& Cognition, 26, 97-107.

Simmering, V. R., \& Spencer, J. P. (2007). Carving up space at imaginary joints: Can people mentally impose arbitrary spatial category boundaries? Journal of Experimental Psychology: Human Perception \& Performance, 33, 871-894. doi:10.1037/0096-1523.33.4.871

SPencer, J. P., \& HUND, A. M. (2002). Prototypes and particulars: Geometric and experience-dependent spatial categories. Journal of Experimental Psychology: General, 131, 16-37. doi:10.1037/0096 $-3445.131 .1 .16$

Spetch, M. L. (1995). Overshadowing in landmark learning: Touchscreen studies with pigeons and humans. Journal of Experimental Psychology: Animal Behavior Processes, 21, 166-181. doi:10.1037/0097 $-7403.21 .2 .166$

Spetch, M. L., \& Kelly, D. M. (2006). Comparative spatial cognition: Processes in landmark- and surface-based place finding. In E. A. Wasserman \& T. R. Zentall (Eds.), Comparative cognition: Experimental explorations of animal intelligence (pp. 210-228). New York: Oxford University Press.

Verbeek, E., \& Spetch, M. (2008). Distortions in location memory. Psychonomic Bulletin \& Review, 15, 328-336. doi:10.3758/ PBR.15.2.328

Wedell, D. H., Fitting, S., \& Allen, G. L. (2007). Shape effects on memory for location. Psychonomic Bulletin \& Review, 14, 681-686.

(Manuscript received June 3, 2009;

revision accepted for publication September 3, 2009.) 